\title{
Bulgarian cystic fibrosis Pseudomonas aeruginosa isolates: antimicrobial susceptibility and neuraminidase-encoding gene distribution
}

Cystic fibrosis (CF) is an autosomal recessive disorder affecting multiple organ systems. The leading cause of morbidity and mortality is a progressive decline in pulmonary function resulting from airway damage caused by thickened secretions, complicated by chronic microbial infection. Pseudomonas aeruginosa is the most prevalent organism colonizing the lungs of CF patients (up to $80 \%$ of the colonizations of lungs of patients aged $>18$ years) (Ramsey, 1996; CFF, 2002). It is rarely eradicated after colonization, regardless of the antimicrobial chemotherapy employed (Govan \& Deretic, 1996). An extracellular neuraminidase is thought to play an important role in $P$. aeruginosa implantation of CF respiratory epithelium (Davies et al., 1999). Nevertheless, the bulk of clinical opinion supports the view that antibiotic therapy leads to improvement in lung function and improves survival of patients, particularly if aggressive treatment is begun on the first isolation of the organism.

The aim of this study was to determine the antimicrobial susceptibility and neuraminidase-encoding gene (nan1) distribution among $P$. aeruginosa isolates recovered from the sputum of CF patients from Bulgaria. A total of 102 P. aeruginosa isolates (including 87 mucoid and 15 nonmucoid strains) identified with the BBL enteric/nonfermenter ID system (Becton Dickinson) were studied. The strains were isolated between December 2005 and April 2008 from 50 patients with CF (30 females and 20 males) aged 6-27. The CF patients came from 17 towns and villages scattered across Bulgaria. The following mutations were found within the cystic fibrosis transmembrane conductance regulatorencoding gene ( $c f t r)$ of the studied patients: $\Delta \mathrm{F} 508 / \Delta \mathrm{F} 508(n=20)$, unknown/ $\Delta$ F508 $(n=6), \mathrm{N} 1303 \mathrm{~K} / \Delta \mathrm{F} 508(n=6)$, unknown/N1303K ( $n=2), \mathrm{R} 1070 \mathrm{Q} /$
218insA $(n=2), \Delta$ F508/R1070Q $(n=2)$, $\Delta$ F508/306delTAGA $(n=2)$, G1069R + L88X/ $\Delta$ F508 $(n=2)$, Q220X/ 2183G-A $(n=2)$, unknown/2184ins108n $(n=2)$ and unknown $(n=4)$. The susceptibilities of the investigated $P$. aeruginosa isolates to 17 antimicrobial agents were determined by the agar dilution method on Mueller-Hinton II agar plates (Becton Dickinson) according to the Clinical and Laboratory Standards Institute (CLSI) 2005 recommendations (CLSI, 2005).

In an attempt to evaluate the evolution of pulmonary disease, we searched for nan1 among the studied isolates of $P$. aeruginosa (Lanotte et al., 2004) using PCR. Total DNA was extracted by boiling. PCR amplification was performed with specific primers nan 1-F (5'-ATGAATACTTATTTTGATA-3') and nan 1-R (5'-CTAAATCCATGCTCTGACCC-3' ${ }^{\prime}$ ) yielding a 1317 bp product (Strateva, 2008). The PCR was carried out with $2 \mu$ template DNA, $0.25 \mu \mathrm{M}$ each primer (Alpha DNA), $0.2 \mathrm{mM}$ dNTPs, $1 \times$ reaction buffer, $2 \mathrm{mM} \mathrm{MgCl}_{2}$ and $1.5 \mathrm{U}$ Prime Taq DNA polymerase (GeNet Bio) in a total volume of $25 \mu \mathrm{l}$. The following PCR protocol was used: an initial denaturation $\left(95^{\circ} \mathrm{C}\right.$, $4 \mathrm{~min}$ ), followed by 25 cycles of denaturation $\left(95{ }^{\circ} \mathrm{C}, 35 \mathrm{~s}\right)$, annealing $\left(53{ }^{\circ} \mathrm{C}, 1 \mathrm{~min}\right)$ and extension $\left(72{ }^{\circ} \mathrm{C}\right.$, $1 \mathrm{~min} 30 \mathrm{~s})$, and a single final extension $\left(72{ }^{\circ} \mathrm{C}, 7 \mathrm{~min}\right)$. Selected PCR products were purified with an ExoSAP-IT reagent (Amersham Biosciences). Sequencing reactions were performed using a sequencing primer, nan $1-\mathrm{F}$-seq (5'-GGTATTTGGAGTGCGCCTA-3')

(Strateva, 2008), the primers for amplification and a BigDye terminator v.3.1. kit (Applera) with an automated sequencer (ABI 310 genetic analyzer; Applied Biosystems). The nucleotide and deduced amino acid sequences were analysed with software available from the
National Center for Biotechnology Information (http://www.ncbi.nlm. nih.gov). The sequence analysis revealed total identity with the $P$. aeruginosa neuraminidase-encoding gene (GenBank accession no. AF236853). Statistical analysis was performed by Student's $t$ test.

The antimicrobial susceptibility testing results are presented in Table 1. A total of 68 of the $102(66.7 \%)$ CF P. aeruginosa isolates studied manifested susceptibilities to all antipseudomonal agents ('wild-type' phenotype). There were slight resistance rates to the following antibiotics: imipenem (5.9\%), meropenem (3.9\%), amikacin $(3.9 \%)$, gentamicin $(7.8 \%)$, tobramycin $(5.9 \%)$, netilmicin $(13.7 \%)$ and ciprofloxacin $(13.7 \%)$. All strains were susceptible to colistin (MICs ranged from 0.03 to $2 \mathrm{mg} \mathrm{l}^{-1}$ ). Colistin-resistant CF isolates of $P$. aeruginosa have been found in some European countries, such as Spain (2.9\%) (Morosini et al., 2005) and the UK (3.1\%) (Pitt et al., 2003). Recently, Johansen et al. (2008) reported two outbreaks and a prolonged spread of colistin-resistant non-mucoid $P$. aeruginosa among chronically infected Danish CF patients. The CF P. aeruginosa isolates from Europe were more susceptible than those isolates from the USA from the same time period. For example, the antibiotic resistance rates in Spain (2003-2004), the UK (2000) and the USA (2000) were, respectively: piperacillin - 20.9, 29.7 and 48.2 \%; ceftazidime - 14.6, 39.6 and $49.9 \%$; tobramycin - 13.1, 10.1 and $22.2 \%$; gentamicin $-33.0,47.0$ and $52.4 \%$; ciprofloxacin - 28.6, 29.7 and $37.4 \%$ (Morosini et al., 2005; Pitt et al., 2003; Burns et al., 2000). The antimicrobial susceptibility of our CF $P$. aeruginosa strains was higher than that of the analogous strains from western Europe and the USA during the last few years, which was related to the predominant stable clinical status of patients 
Table 1. Antimicrobial susceptibility of 102 isolates of $P$. aeruginosa from Bulgarian CF patients

\begin{tabular}{|c|c|c|c|}
\hline \multirow[t]{2}{*}{ Antimicrobial agent } & \multicolumn{3}{|c|}{ No. (\%) of isolates } \\
\hline & Susceptible & Intermediate & Resistant \\
\hline Carbenicillin & $98(96.1)$ & $2(2)$ & $2(2)$ \\
\hline Azlocillin & $102(100)$ & $0(0)$ & $0(0)$ \\
\hline Piperacillin & $102(100)$ & $0(0)$ & $0(0)$ \\
\hline Piperacillin + tazobactam & $102(100)$ & $0(0)$ & $0(0)$ \\
\hline Ceftazidime & $102(100)$ & $0(0)$ & $0(0)$ \\
\hline Cefoperazone & $102(100)$ & $0(0)$ & $0(0)$ \\
\hline Cefepime & $98(96.1)$ & $2(2)$ & $2(2)$ \\
\hline Cefpirome & $98(96.1)$ & $2(2)$ & $2(2)$ \\
\hline Aztreonam & $98(96.1)$ & $2(2)$ & $2(2)$ \\
\hline Imipenem & $94(92.1)$ & $2(2)$ & $6(5.9)$ \\
\hline Meropenem & $98(96.1)$ & $0(0)$ & $4(3.9)$ \\
\hline Amikacin & $98(96.1)$ & $0(0)$ & $4(3.9)$ \\
\hline Gentamicin & $90(88.2)$ & $4(3.9)$ & $8(7.8)$ \\
\hline Tobramycin & $96(94.1)$ & $0(0)$ & $6(5.9)$ \\
\hline Netilmicin & $86(84.3)$ & $2(2)$ & $14(13.7)$ \\
\hline Ciprofloxacin & $84(82.3)$ & $4(3.9)$ & $14(13.7)$ \\
\hline Colistin & $102(100)$ & $0(0)$ & $0(0)$ \\
\hline
\end{tabular}

Table 2. Characteristics of the CF patients $(n=14)$ who had $P$. aeruginosa isolates expressing a neuraminidase-encoding gene

\begin{tabular}{|lc|}
\hline Characteristic & Value \\
\hline Age & \\
Mean & 13.4 years \\
Range & $6-17$ years \\
Sex & \\
Male & 4 patients \\
Female & 10 patients \\
Patient symptom & \\
Increased cough & 14 patients \\
Increased sputum production & 14 patients \\
Dyspnoea & 6 patients \\
Weight loss & 9 patients $($ loss in range $1-4 \mathrm{~kg})$ \\
Temperature range & $37.6-39.4{ }^{\circ} \mathrm{C}$ \\
New auscultation findings & In all patients \\
Sputum cytology & \\
PNL range & $29-68 \%$ \\
Metaplasia range & $0-70 \%$ \\
Complete blood count & \\
WBC range & $7.10-19.34 \times 10^{9}$ cells $1^{-1}$ \\
Neu range & $51.40-83.16 \%$ \\
ESR range & $17-50$ mm h ${ }^{-1}$ \\
CRP serum concentration range & $3.9-7.2$ mg $1^{-1}$ \\
X-ray & \\
Pneumonia & 12 patients \\
Emphysema & 11 patients \\
Spirometry & \\
Mean FEV ${ }_{1}$ at baseline as percentage of predicted value & (range $49-82 \%)$ \\
Mean FEV ${ }_{1}$ on day of exacerbation as percentage of predicted value & \\
& \\
\hline
\end{tabular}

CRP, C-reactive protein; ESR, erythrocyte sedimentation rate; Neu, neutrophil granulocytes; PNL, polynuclear leukocytes; WBC, white blood cells.

${ }^{\star}$ Here are noted only newly found changes, compared to the previous X-ray of the patient; we didn't count the old changes from previous exacerbations. 
investigated, and thus the lack of prolonged antimicrobial chemotherapy. On auscultation, most patients had a clear vesicular murmur (with bronchial character in the places where bronchiectasis was formed) without any crackles (rales) or rhonchi. All data from complete blood counts were within normal limits or on the upper limit. There were no additional new changes on X-ray findings. The spirometry showed steady levels of forced vital capacity and forced expiratory volume in $1 \mathrm{~s}\left(\mathrm{FEV}_{1}\right)$ slightly decreasing with the age of the patient. The sputum cytology manifested steady levels of polynuclear cells and the rate of metaplasia was not increasing significantly with check-ups. The patients in good clinical condition usually needed an antibiotic added to their regular treatment once or a maximum of twice yearly.

As has been described elsewhere, the prevalence of nan1 in CF isolates has tended to increase as the clinical status worsened (Lanotte et al., 2004). In this research a total of 39 of the 102 CF $P$. aeruginosa isolates expressed a nanl gene. The nan1-positive strains of $P$. aeruginosa $(38.2 \%)$ were isolated from 14 patients with recurrent exacerbations of pulmonary disease and a weak clinical status. The characteristics of these patients are summarized in Table 2. Exacerbations were characterized by acute changes in respiratory signs and symptoms, such as increased cough frequency and sputum production, weight loss, new auscultation findings (crackles, wheezing, rales or rhonchi), inflammation changes according to the complete blood count (elevated leukocytes, erythrocyte sedimentation rate and C-reactive protein), new changes on $\mathrm{X}$-ray and elevated levels of polynuclear cells and metaplasia on sputum cytology. In addition, the drop in forced vital capacity and $\mathrm{FEV}_{1}$ was drastic, and hardly came back to the baseline after the treatment.

The frequency of nan 1 in our study (38.2\%) was significantly lower than that among recent French CF isolates of $P$. aeruginosa $(61.7 \%)(P<0.001)$ (Lanotte et al., 2004). The different frequencies of nan1 among Bulgarian and French CF patients resulted from the clinical status of the patients.
There were also some features of the antibiotic policy in Bulgaria that contributed to the high antimicrobial susceptibility in CF $P$. aeruginosa isolates, such as an empirical therapy of $P$. aeruginosa colonization/infection predominantly with oral ciprofloxacin, difficulties in the supply of inhalatory therapy with tobramycin, because of the high price and the necessity of prolonged use of the drug (28 day courses), and a lack of a licence for the clinical use of colistin in Bulgaria.

The predominant portion $(85.3 \%)$ of our CF strains were mucoid. Traditionally mucoid $P$. aeruginosa isolates have higher resistance and long-term colonization, but there are enough scientific reports describing more susceptible CF mucoid $P$. aeruginosa strains compared with nonmucoid strains (Ciofu et al., 2001; Srifuengfung et al., 2004).

In conclusion, the antimicrobial resistance of Bulgarian CF P. aeruginosa isolates was unusually low, which was related to the following causes: mostly intermittent and chronic colonization/infection without leaning towards recurrent exacerbations in the studied patients, and thus a lack of prolonged antimicrobial chemotherapy; and the antibiotic policy in Bulgaria. Also, the frequency of nan 1 was relatively low, and correlated with the predominant good clinical status of the patients investigated. The molecular-genetic detection of this gene may be used as an indirect measure of CF pulmonary disease evolution.

\section{Tanya Strateva, ${ }^{1}$ Guergana Petrova, ${ }^{2}$ Penka Perenovska ${ }^{2}$ and Ivan Mitov ${ }^{1}$}

\section{${ }^{1}$ Department of Microbiology, Medical University of Sofia, 2 Zdrave Street, 1431 Sofia, Bulgaria \\ ${ }^{2}$ Alexandrovska University Hospital, Medical University of Sofia, Sofia, Bulgaria}

Correspondence: Tanya Strateva (dr.strateva@abv.bg)

Burns, J. L., Saiman, L., Whittier, S., Larone, D., Krzewinski, J., Liu, Z., Marshall, S. A. \& Jones, R. N. (2000). Comparison of agar diffusion methodologies for antimicrobial susceptibility testing of Pseudomonas aeruginosa isolates from cystic fibrosis patients. J Clin Microbiol 38, $1818-1822$
Ciofu, O., Fussing, V., Bagge, N., Koch, C. \& Høiby, N. (2001). Characterization of pared mucoid/non-mucoid Pseudomonas aeruginosa isolates from Danish cystic fibrosis patients: antibiotic resistance, $\beta$-lactamase activity and ribotyping. J Antimicrob Chemother 48, 391-396.

CLSI (2005). Performance Standards for Antimicrobial Susceptibility Testing, 15th informational supplement, M100-S15. Wayne, PA: Clinical and Laboratory Standards Institute.

CFF (2002). Patient Registry 2001 Annual Data Report. Bethesda, MD: Cystic Fibrosis Foundation.

Davies, J., Dewar, A., Bush, A., Pitt, T., Gruenert, D., Geddes, D. M. \& Alton, E. W. (1999). Reduction in the adherence of Pseudomonas aeruginosa to native cystic fibrosis epithelium with anti-asialoGM1 antibody and neuraminidase inhibition. Eur Respir J 13, 565570.

Govan, J. R. \& Deretic, V. (1996). Microbial pathogenesis in cystic fibrosis: mucoid Pseudomonas aeruginosa and Burkholderia cepacia. Microbiol Rev 60, 539-574.

Johansen, H. K., Moskowitz, S. M., Ciofu, O., Pressler, T. \& Høiby, N. (2008). Spread of colistin resistant non-mucoid Pseudomonas aeruginosa among chronically infected Danish cystic fibrosis patients. J Cyst Fibros 7, 391-397.

Lanotte, P., Watt, S., Mereghetti, L., Dartiguelongue, N., Rastegar-Lari, A., Goudeau, A. \& Quentin, R. (2004). Genetic features of Pseudomonas aeruginosa isolates from cystic fibrosis patients compared with those of isolates from other origins. J Med Microbiol 53, 73-81.

Morosini, M. I., Garcia-Castillo, M., Loza, E., Perez-Vazquez, M., Baquero, F. \& Canton, R. (2005). Breakpoints for predicting Pseudomonas aeruginosa susceptibility to inhaled tobramycin in cystic fibrosis patients: use of high-range E-test strips. J Clin Microbiol 43, 4480-4485.

Pitt, T. L., Sparrow, M. \& Stefanidou, M. (2003). Survey of resistance of Pseudomonas aeruginosa from UK patients with cystic fibrosis to six commonly prescribed antimicrobial agents. Thorax 58, 794-796.

Ramsey, B. W. (1996). Management of pulmonary disease in patients with cystic fibrosis. N Engl J Med 335, 179-188.

Srifuengfung, S., Tiensasitorn, C., Yungyuen, T. \& Dhirapurta, C. (2004). Prevalence and antimicrobial susceptibility of Pseudomonas aeruginosa mucoid and non-mucoid type. Southeast Asian J Trop Med Public Health 35, 893-896.

Strateva, T. (2008). Microbiological and molecular-genetic investigations on the resistance mechanisms and virulence factors in clinical strains of Pseudomonas aeruginosa. $\mathrm{PhD}$ thesis, Medical University of Sofia. 